\title{
X-ray Characterization of Internal and Near-nozzle Flow from a Standard Simplex Atomizer Spray
}

\author{
Brandon A. Sforzo*, Alan L. Kastengren, Jan llavsky, Christopher F. Powell \\ Argonne National Laboratory, 9700 S. Cass Ave, Lemont, IL 60439, USA \\ ${ }^{*}$ Corresponding author: bsforzo@anl.gov
}

\begin{abstract}
Fluid flow and atomization from two standardized simplex injectors was examined using a set of $\mathrm{x}$-ray diagnostic techniques. X-ray phase contrast imaging was used to visualize the filming behavior of the liquid inside the swirl chamber, revealing the air core and liquid film profile within the metal nozzle. Ultra-small angle X-ray scattering (USAXS) was used to quantify the atomization behavior of the external spray. Specifically, the path-specific surface area of the spray was collected for transverse profiles of the hollow-cone at several downstream distances. This result highlights the sheet disintegration as well as the evolution of the fluid breakup. Furthermore, liquid volume measurements from prior projected mass measurements was combined with the USAXS results to generate characteristic Sauter mean diameter profiles for each of the injectors and conditions at several locations in the spray.
\end{abstract}

\section{Keywords}

X-ray, Diagnostics, USAXS, Simplex, Pressure-swirl

\section{Introduction}

Pressure-swirl atomizers are found across a wide range of applications where liquid dispersion and mixing with the surroundings is required. These injectors are used in industrial, agricultural, and propulsion spray settings, owing their adoption to the simple design and ability to disperse liquid at wide angles. However, increasing flow requirements requires a disproportionate increase in supply pressure, and varying supply pressure can cause operation to switch between liquid breakup mechanisms. Predicting this operational behavior requires accurate models when designing hardware for new applications. Pursuit of a standardized spray was previously executed by McDonell et al. [1] to collect a comprehensive set of interlaboratory data as a reference for building such improved models. Improved upstream prediction of the liquid flow requires additional validation in these regions.

Here, x-ray diagnostics are applied to the hardware and sprays of the standard simplex atomizers to examine internal and near-nozzle phenomena [2]. As stated by previous modeling works, the internal flow and air core formation in the simplex nozzle is critical in determining the flow area and discharge behavior and affects all downstream performance [3]. Theoretical frameworks have been established for the general internal flow within a simplex atomizer [4]. These models lead to predictions of film thickness and sheet development, which are in turn related to droplet sizes [5], but use of these models necessitates confirmation of the air core behavior. Experimental works have examined internal flows within general pressure-swirl atomizer geometries through the construction of transparent models $[6,7,8]$. These studies provide parametric understanding on the influence of operating condition to the internal flow and the external spray behavior. Similar validation is desired for as-built hardware, specific to the multi-institutional investigations of the standardized design.

The ultimate goal of this work is to build upon the existing studies of the standardized Research Simplex Atomizers carried out by Thistle et al. [9], Leask et al. [10], Sforzo et al. [11], and Jacobsohn et al. [12]. First, x-ray imaging will be presented of the internal flow structure of each simplex atomizer to characterize the air core and developing liquid sheet. Secondly, path-specific surface area values will be computed from ultra-small angle x-ray scattering (USAXS) measurements. Ultra-small-angle $x$-ray scattering has been matured in recent years as a method of quantifying the near-nozzle atomization quality [13] and is applied here to characterize the interfacial surface area of the atomizing flowfield. Combined with previously collected absorption measurements will yield a Sauter mean diameter (SMD) quantification in the sprays of interest.

\section{Methodology}

Two Research Simplex Atomizer injectors and the sprays they produced were studied with x-ray diagnostic methods at the Advanced Photon Source (APS) at Argonne National Laboratory. These injectors, produced for spray and combustion studies with practical industry methods and materials, labeled "SN005" and "SN010" have been compared and analyzed previously $[10,11,12]$. As in these previous studies, the injectors were supplied with water via a dip-tube tank, pressurized using gaseous nitrogen. The atomizers were also mounted such that the spray injected downward to replicate the conditions and methods carried out in the prior studies. Here, the nominal supplied fluid pressure was set to either $345 \mathrm{kPa}(50 \mathrm{psi})$ or $690 \mathrm{kPa}(100 \mathrm{psi})$ as measured immediately upstream of the injector housing. The injector design, as tested here, is presented in Fig. 1, to illustrate the nominal internal features.

\section{Imaging}

High speed x-ray imaging was performed at the 7-ID beamline at the APS [14]. As illustrated in Fig. 2, the polychromatic (white) x-ray beam, originating from an APS Undulator A source with a $14 \mathrm{~mm}$ gap, was sized to a 


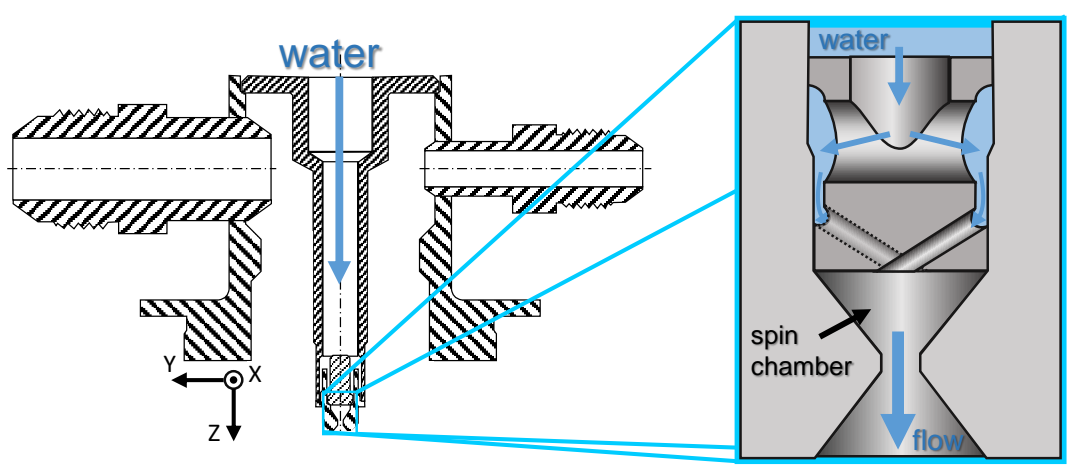

Figure 1. Coordinate system of simplex atomizers. Inset schematic details the nominal internal geometry as provided by Ref. [9]

$2.50 \times 2.50 \mathrm{~mm}(\mathrm{H} \times \mathrm{V})$ rectangular cross-section with beam defining slits. A mechanical chopper was used to temporally gate the incoming beam and limit thermal loading on the hardware. As the beam passed through the operating injector, the $x$-rays were both attenuated and slightly refracted, proving contrast intensity variations at the phase boundaries. The transmitted beam was incident on a LuAG:Ce scintillator, which fluoresced in visible light. This light was then imaged using a 5x microscope objective, coupled to a FLIR Grasshopper3, $4096 \times 3000 \mathrm{px}$ CMOS camera, resulting in a native imaging resolution of $0.664 \mu \mathrm{m} \cdot \mathrm{px}^{-1}$. The regions of interest in the focusing effort were confined to the internal observation of the geometry and flow features.

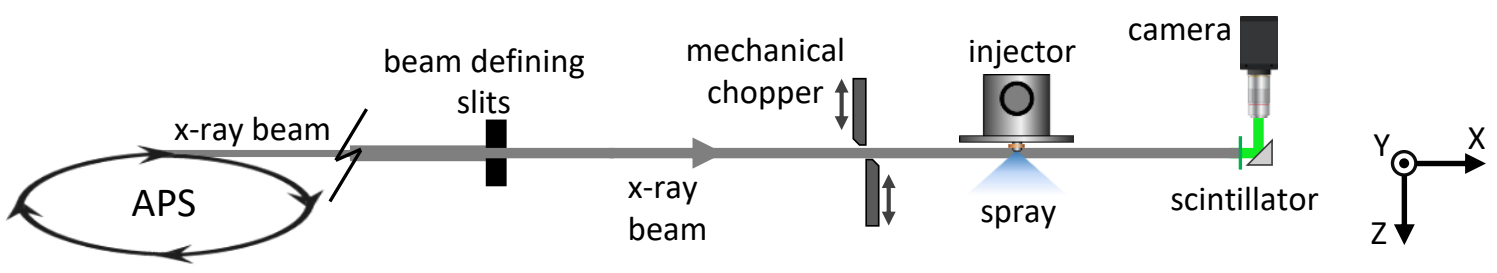

Figure 2. Layout of imaging configuration.

\section{Ultra-small-angle $X$-ray Scattering}

USAXS measurements were carried out at the 9-ID beamline [15] of the APS, using a Bonse-Hart instrument as shown in Fig. 3. In this instrument, the incoming $x$-ray light has been conditioned to a photon energy of $21 \mathrm{keV}(\lambda$ $=0.59 \AA)$, and profiled to $200 \times 200 \mu \mathrm{m}(\mathrm{V} \times \mathrm{H})$ with beam-defining slits. The $x$-rays are precisely collimated with pair of $\mathrm{Si}$ (220) crystals and the beam intensity is measured with an ion chamber. The beam then passes into the spray, scattering the photons at small angles. A pair of analyzer Si (220) crystals were effectively used as an angle filter. They were scanned in angle probe the scattered $x$-ray intensity as a function of angle.

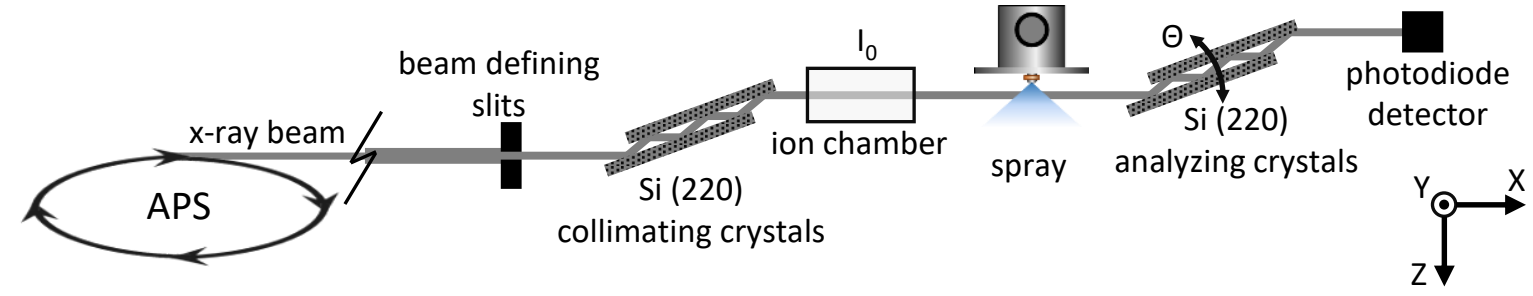

Figure 3. Layout of the USAXS experiment.

To analyze the USAXS data, the scattered x-ray intensity as a function of angle is converted to differential cross section $(d \Sigma / d \Omega)$ as a function of scattering vector $(q)$. The droplet sizes in the current study are much larger than the inverse of the smallest scattering vector that can effectively be probed with the USAXS instrument. While this precludes a typical SAXS analysis of scattering curve shapes to understand particle size, in this regime one can use Porod's Law [16], as defined by:

$$
\frac{d \Sigma}{d \Omega}(q)=2 \pi \Delta \rho^{2} S q^{-4}
$$




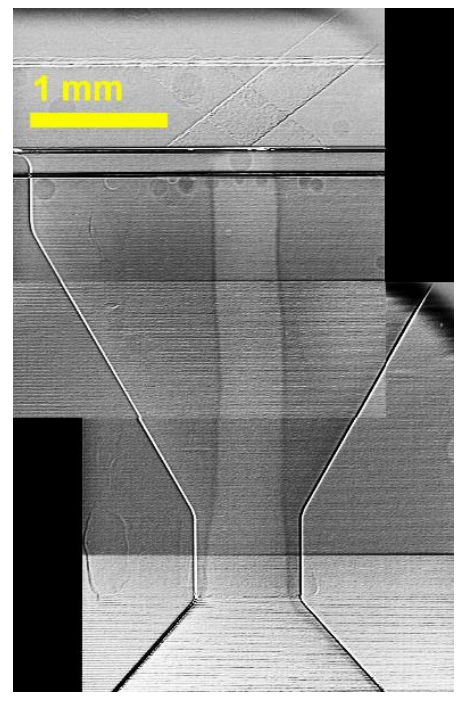

SN005, $690 \mathrm{kPa}$

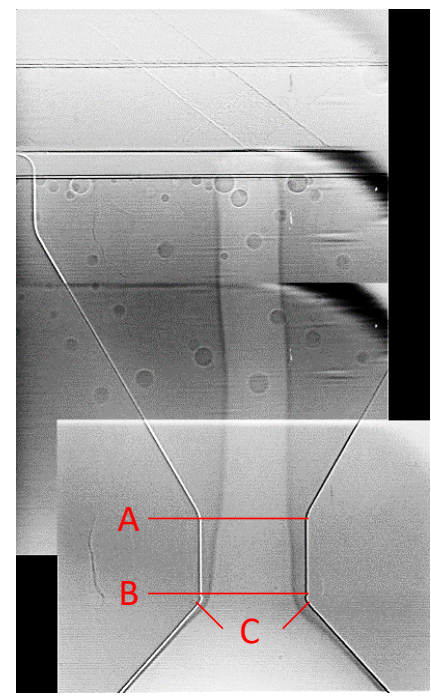

SN005, $345 \mathrm{kPa}$

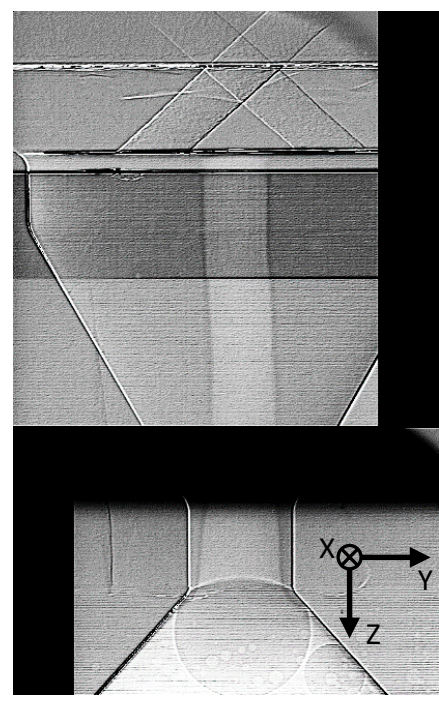

SN010, $690 \mathrm{kPa}$

Figure 4. X-ray imaging of the internal flow of the simplex atomizer, visualizing the inner aid vortex core and liquid flow on the prefilming surface. Dark circular artifacts are due to static liquid droplets appearing on the exterior surface or internal orifice when the spray is off and the flat field images were acquired.

where, $S_{p}$ is the surface area per beam area, and $\Delta \rho$ is the scattering length contrast of the particles compared to the surrounding medium. This provides a measurement of the specific surface area of the spray. This relationship assumes that the particles are homogeneous, relatively dilute, and locally non-interacting. Furthermore, it is assumed that the particles are randomly oriented which is expected to hold for this water spray after the sheet breakup region. The spray surface area was determined for each probe volume where full USAXS curves were gathered by using the Irena software for fitting the Porod model [17]. It should be noted that these specific surface area data are pathlength integrated, and as such represent a projection of the 3D distribution of surface area in the spray onto a 2D measurement plane.

\section{Results and discussion Internal Flow}

Each injector was operated with supply pressure of $690 \mathrm{kPa}$, and the SN005 injector was operated at $345 \mathrm{kPa}$ for imaging of the internal flow. Due to the thickness and composition of the atomizer hardware material, time-resolved observation of the liquid flow inside the injector was not possible. For each viewing region of interest, the camera acquired 10 images with no flow, and 50 images with flow. Framing was performed at $1 \mathrm{~Hz}$, with a $1 \mathrm{~ms}$ exposure, in order to achieve mid-range intensity on the detector for each frame. The median image of each sequence was taken (as opposed to the mean) in order to remove bright pixels caused by scattered $\mathrm{x}$-rays absorbed in the visible cameara sensor. The data frames were then normalized by the flat-field images to visualize the internal flow. The $\mathrm{ROI}$ frames were assembled into a mosaic for each condition, which are presented in Fig. 4. In these three mosaics, three to four ROls were collected to capture the spin chamber region of the internal geometry. In the SN010 mosaic, presence of a spherical droplet at the outlet of the nozzle is an artifact of the presence of a bubble in the flat field image. Additionally, circular dark artifacts are visible in the SN005 images which are caused by static liquid droplets that were present on the exterior of the injector during the flat-field image acquisition.

In each of these mosaics, the persistent phase boundary between the flowing water and air core is prominently visible. In all cases, the air core extends entirely up to the internal inlet surface of the spin chamber, and appearing, qualitatively, similar in these cases. The air core boundaries have some minor curvature in the upper regions, indicating potential asymmetries in the internal flow in the swirl chamber. The air core flares outward slightly (thinning of the liquid sheet) as the spin chamber converges toward the nozzle. At the throat of the nozzle, the liquid sheet appears to have additional undulations in the surface, and the thickness of the film differs on each side of the nozzle. The evolution of the apparent liquid thickness are depicted in Fig. 5.

The line plots illustrate that the liquid film for both sides in all three cases exit the converging section and enter the nozzle at roughly the same thickness. However, from this point, the behavior for each side and between the cases diverge. Possibly due to an asymmetry in the geometry of the injectors, the film is thinner on the $-Y$ side of the nozzle in all cases. Furthermore, for the SN005 nozzle the mean film thickness at location C for $690 \mathrm{kPa}$ injection pressure is $56 \mu \mathrm{m}$, while for the same injector at $345 \mathrm{kPa}$ injection pressure, this average thickness only increases to $59 \mu \mathrm{m}$. For the SN010 injector, the average thickness at location C is $47 \mu \mathrm{m}$. Though this is not the outlet of the hardware into the free spray region, it is expected that the film thickness is proportional to the mean droplet size in the spray [18]. 


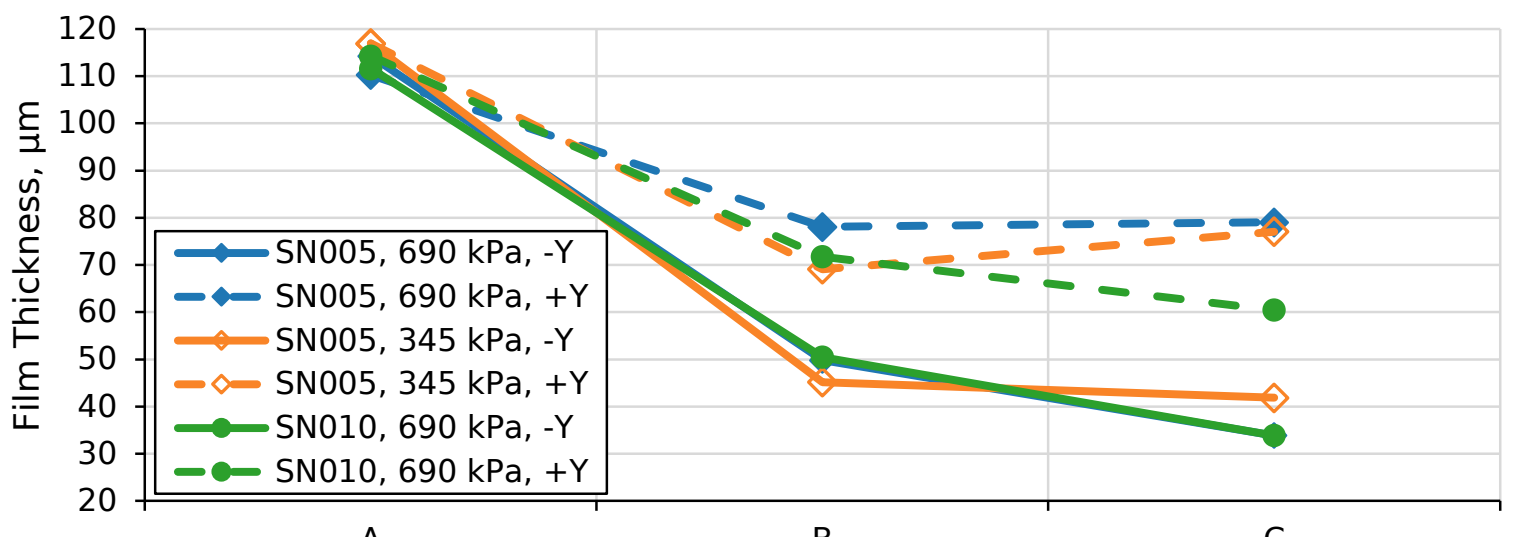

A

B

C

Axial Location

Figure 5. Comparison of the film thickness evolution measured on each side of the imaged nozzles. Measured locations are depicted in Figure 4. Note that the thickness evolution for "SN005,690 kPa, $-Y^{\prime}$ " falls directly under the profile for "SN010,690 kPa, $-Y^{\prime \prime}$.

\section{Near-Nozzle Atomization Performance}

Atomization behavior was investigated through the collection of USAXS measurements. As pioneered for spray measurements by previous works, spray surface area measurements were simplified by collecting transverse scans of scattered $x$-ray light at a fixed q (scattering vector) $[19,13]$. The intensities of these scans were scaled proportionally to the computed path-specific surface areas $\left(S_{p}\right)$ determined from full USAXS curves collected from a subset of points in each transverse scan. Transverse and axial scans to the spray were collected for both injectors and at both 345 and $690 \mathrm{kPa}$ liquid injection pressure. Transverse scans at several axial (Z) distances from each injector for the $690 \mathrm{kPa}$ supply pressure case are compiled into a 2D mapping and presented in Fig. 6 .

For both nozzles, the transverse scans are bimodal, which is logical given that the liquid distribution is annular and these data are a projection of the surface area distribution. Values generally decrease with increasing Z, which is tied to the rapid reduction in liquid pathlength as the spray spreads. As previously observed [11], the SN005 injector has asymmetric behavior, and this can be seen by the evolution in scattering profiles, specifically with a ridge in scattering, possibly caused by a persistent ripple in the liquid sheet. These patterns may originate in the internal flow, as imaged above, evidenced by the asymmetric film thickness, which is linked to the microscopic geometry features of the nozzle $[11,12]$. However, while the asymmetry of the internal liquid film is also observed for the SN010 injector, the scattering profile has evolved much more uniformly, suggesting that the disturbance could be transient or additional factors influence as the flow continues to develop.

The SN010 injector spray exhibits symmetric scattering profiles. Along the SN010 spray axis, the scattering is at a maximum at the nozzle exit and decreases until $Z=3 \mathrm{~mm}$ where it locally increases, and eventually levels to $S_{p}=0.75 \mathrm{~mm}^{2} / \mathrm{mm}^{2}$. However, along the edges of the spray, where the scattering intensity is highest, the signal increases until $Z=2.5 \mathrm{~mm}$ and then monotonically decreases, leveling to $S_{p}=1 \mathrm{~mm}^{2} / \mathrm{mm}^{2}$.

In addition to transverse (Y-dimension) scans at many Z-positions, individual axial scans at $Y=0$ were collected. These data shared common probe locations with absorption measurements previously gathered, which report the projected mass $(M)$ [11]. These axial profiles of projected mass are plotted on the left chart of Figure 7, and illustrate a monotonically decreasing value, progressing away from the nozzle, for all cases. Similar to the transverse scans, the scattering measurements collected along the axial scan were scaled based on individual USAXS curves collected and fit with Porod's model, with a weighting toward the downstream region where the droplets were locally less interacting. The axial profile of path-specific surface area for each condition is presented in the center chart of Fig. 7. Similar to the projected mass, there is a near-nozzle decrease in scattering, and therefore surface area, until $Z=3 \mathrm{~mm}$ where it locally increases. This suggests the location of the sheet breakup, where the ratio of liquid mass to interfacial surface area are changing.

The radiography results, in combination with the water density, result in liquid volume. This liquid volume is combined with the $S_{p}$ values from the same measurement locations to yield the calculated Sauter mean diameter (SMD, $\left.d_{32}\right)$, via an established formulation [20, 21]. The relation,

$$
d_{32}=6 \frac{V}{S_{p}},
$$

was used, where $S_{p}$ was collected through the USAXS measurements and $V$ was obtained from radiography. The SMD values along the axial centerline for all conditions, are presented in the right chart of Fig. 7 . This ratio indicates the breakup region where the ratio of liquid volume to surface area is rapidly changing. Furthermore, these profiles suggest that for the SN010 injector at both conditions, the SMD values converge to similar values beyond Z=7.5 mm, approximately $110 \mu \mathrm{m}$. The SN005 injector, at $690 \mathrm{kPa}$, converged to a lower SMD by $Z=10 \mathrm{~mm}$, approximately $80 \mu \mathrm{m}$. 

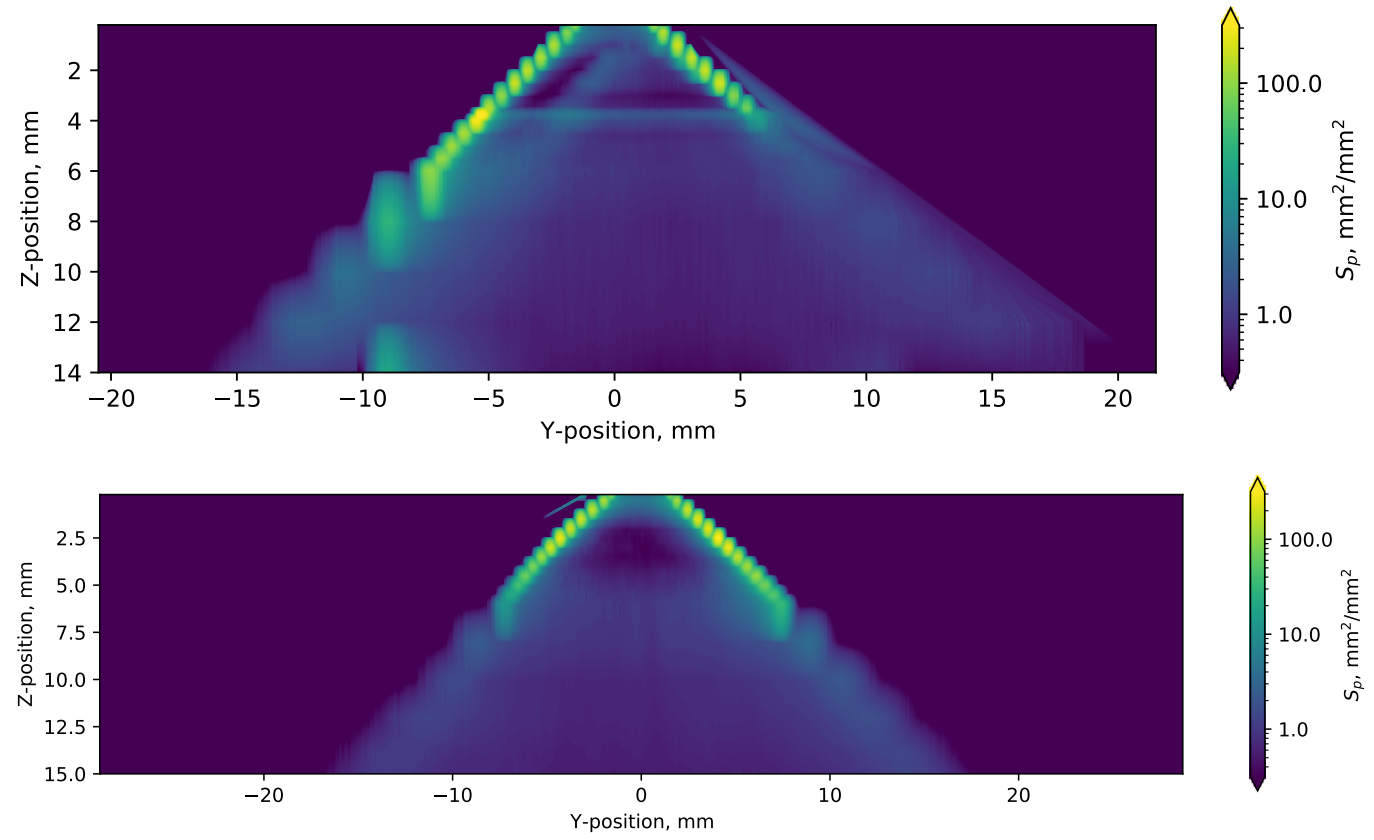

Figure 6. Path specific surface area for multiple transverse scans of sprays from SN005 (top) and SN010 (bottom) injectors, at $690 \mathrm{kPa}$ liquid supply pressure.
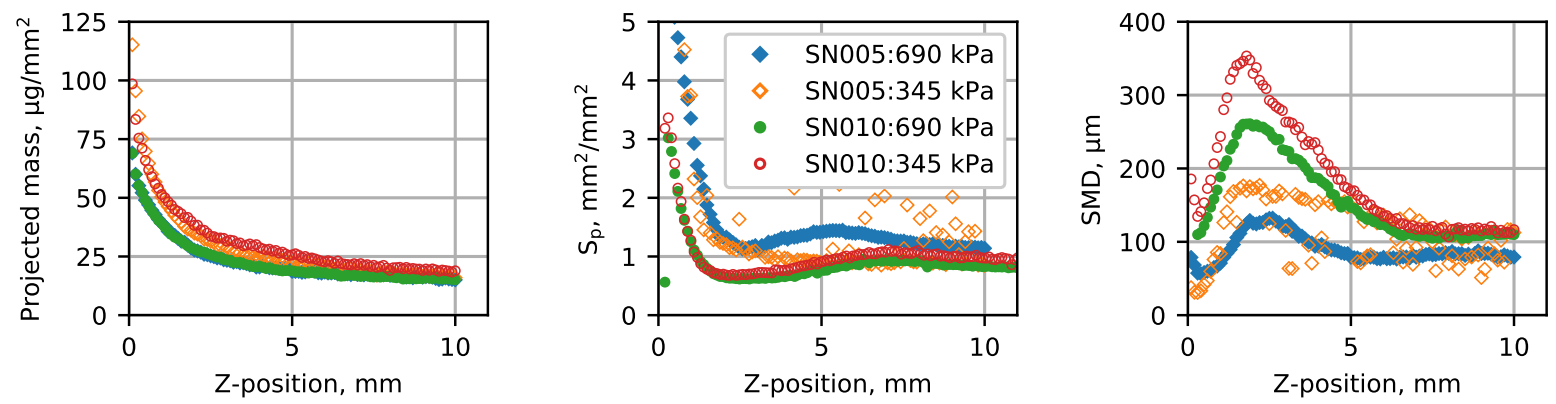

Figure 7. Axial profiles of three metrics of atomization quality for the four conditions: (left) Radiography of the projected mass, (center) path-specific surface area, and (right) Sauter mean diameter.

As observed previously [11], the asymmetric behavior of SN005 was particularly erratic at low supply pressures. The $S_{p}$ profile at the $345 \mathrm{kPa}$ condition contains many outliers when compared to the other conditions. However, when SMD is computed, the values appear to remain contained between the other profiles, suggesting that there is some modal behavior for the injector at this condition, and may not be fully-developed [18].

\section{Summary and Conclusions}

Additional measurements have been made to the Research Simplex Atomizers using x-ray diagnostics. Firstly, x-ray imaging was collected for both injectors under operation at multiple conditions to observe the time-averaged internal flow structure. The phase boundary of the flowing water in the spin chamber highlighted the presence of a persistent air core that extends up to the interior chamber top wall at both 345 and $690 \mathrm{kPa}$ liquid supply pressures. Subtle differences exist for the shape and dimensions of the air core until the exit of the nozzle where the asymmetric film thicknesses diverge for each condition.

The near-field of the sprays of each injector were scanned to collect ultra-small angle x-ray scattering data. These measurements were directly converted into path-specific surface area profiles, illustrating the axial evolution of the spray. This also further revealed the asymmetric behavior of the SN005 injector spray as well as the difference in near-field scattering through vs. tangent to the liquid sheet. Scattering area measurements were compared to the liquid volume measurements previously collected to compute axial SMD profiles for the evolving spray. These values are most relevant at axial distances where the liquid sheet has disintegrated, shown to happen near $Z=5 \mathrm{~mm}$, which coincides with a convergence of SMD value for most cases. For these tests, the SN010 injector spray converged to a similar SMD value for both pressure cases by $Z=10 \mathrm{~mm}$. The linkage between the internal flow in these injectors and the near-field atomization is not clear, which encourages further investigation and analysis of the flow behavior. Specifically, additional time-resolved imaging of the film evolution throughout the internal geometry would 
help clarify the temporal character of the flow. Additionally, comparison of SMD values in the mid-field region would further verify the evolution of the atomization behavior from these injectors.

These observations add to the growing collection of experimental characterization of the Research Simplex Atomizers. This standardized design and the collaborative investigation serves to enrich a database of high-fidelity measurements for the purposes of developing improved predictive models. This contribution will serve to validate internal flow simulations within the chamber as well as primary atomization models of the near-field sheet breakup.

\section{Acknowledgements}

Research presented in this paper was performed at the 7-BM, 7-ID, and 9-ID beamlines at the Advanced Photon Source at Argonne National Laboratory. Use of the APS is supported by the U.S. Department of Energy (DOE) under Contract No. DEAC0206CH11357. The Argonne fuel spray research is sponsored by the DOE Vehicle Technologies Program under the direction of Gurpreet Singh and Michael Weismiller.

\section{References}

[1] VG McDonnell, GS Samuelsen, MR Wang, CH Hong, and WH Lai. Journal of Propulsion and Power, 10(3):402409, 1994.

[2] Alan Kastengren and Christopher F. Powell. Experiments in Fluids, 55(3):1686, Feb 2014.

[3] A. Datta and S.K. Som. International Journal of Heat and Fluid Flow, 21(4):412-419, 2000.

[4] A. M. Binnie and D. P. Harris. The Quarterly Journal of Mechanics and Applied Mathematics, 3(1):89-106, 01 1950.

[5] NK Rizk and Arthur Henry Lefebvre. Journal of Propulsion and Power, 1(3):193-199, May 1985

[6] M.R. Halder, S.K. Dash, and S.K. Som. Experimental Thermal and Fluid Science, 26(8):871-878, 2002.

[7] Seoksu Moon, Choongsik Bae, Essam Abo-Serie, and Jaejoon Choi. Atomization and Sprays, 17(6):529-550, 2007.

[8] Ghobad Amini. International Journal of Multiphase Flow, 79:225-235, mar 2016.

[9] Andrew Thistle, Vincent McDonell, and Benjamin Halls. Standard simplex atomizer program status and expectations. 14th International Conference on Liquid Atomization and Spray Systems. Institute for Liquid Atomization and Spray Systems, Chicago, IL, 2018.

[10] Scott B Leask, Alice K Li, Vincent G McDonell, and Scott Samuelsen. ASME 2018 International Mechanical Engineering Congress and Exposition, pp. V08AT10A024-V08AT10A024. American Society of Mechanical Engineers, 2018.

[11] Brandon Sforzo, Scott Leask, Alice Li, Aniket Tekawade, Christopher Powell, Vincent McDonell, and Alan Kastengren. AIAA Scitech 2020 Forum, 2020.

[12] Gabriel L. Jacobsohn, Brandon Sforzo, Alan Kastengren, Aniket Tekawade, Christopher F. Powell, Scott B. Leask, Alice K. Li, and Vincent G. McDonell. AIAA Scitech 2021 Forum, 2021.

[13] K. E. Matusik, B. A. Sforzo, H. J. Seong, D. J. Duke, A. L. Kastengren, J. Ilavsky, and C. F. Powell. Atomization and Sprays, 2019. Under Review.

[14] Eric M. Dufresne, Bernhard Adams, Dohn A. Arms, Matthieu Chollet, Eric C. Landahl, Yuelin Li, Donald A. Walko, and Jin Wang. AIP Conference Proceedings, 1234(1):181-184, 2010.

[15] Jan Ilavsky, Pete R. Jemian, Andrew J. Allen, Fan Zhang, Lyle E. Levine, and Gabrielle G. Long. Journal of Applied Crystallography, 42(3):469-479, Jun 2009.

[16] Jens Als-Nielsen and Des McMorrow. Elements of modern X-ray physics. John Wiley \& Sons, 2011.

[17] Jan llavsky and Peter R. Jemian. Journal of Applied Crystallography, 42(2):347-353, Apr 2009.

[18] Arthur H Lefebvre and Vincent G McDonell. Atomization and sprays. CRC press, 2017.

[19] K. E. Matusik, B. A. Sforzo, H. J. Seong, D. J. Duke, A. L. Kastengren, J. llavsky, and C. F. Powell. X-ray measurements of fuel spray specific surface area and sauter mean diameter for cavitating and non-cavitating diesel sprays. ICLASS 2018. ILASS Americas, 2018.

[20] A. Kastengren, J. llavsky, Juan Pablo Viera, Raul Payri, D.J. Duke, A. Swantek, F. Zak Tilocco, N. Sovis, and C.F. Powell. International Journal of Multiphase Flow, 92:131 - 139, 2017.

[21] CF Powell, D Duke, AL Kastengren, and J llavsky. 25th ILASS-Americas conference, Pittsburgh, PA, 2013. 